\title{
Cerebirovascular Diseases
}

Acute ischemic stroke 151, 202

- stroke 12,67,355

- - diagnosis 241

- $\quad$, , unclear onset 572

- treatment 457

Adrenomedullin 164

Alcohol intake 517

Angioplasty 401

Anisotropy 209

Anticoagulation, early 289

Anticonvulsants 107

Anti-oxidized low-density-lipoprotein antibody 122

Antiplatelet 344

- therapy 355

Anxiety 311

Aphasia 316

Aspirin 344

- resistance 392

Aspirin-clopidogrel 392

Atherosclerosis 74, 417

Atherothrombosis 366

Atrial fibrillation 344

Automated and visual scoring methods 59

Basilar artery occlusion 511

Bilateral movement training 115

Blood brain barrier 338

- flow 430

- pressure 261

- - control 526

Bone 95

Brain edema 176

- infarction 202

- ischaemia 268

- perfusion SPECT 580

- vascular malformations 157

C593T GPX1 polymorphism 445

Cardioembolic stroke 289

Cardiovascular disease risk 526

- _ _ factors 254

Caregivers 234

Caregiving 548

Carotid and transcranial color-coded duplex ultrasonography 304

- angioplasty stenting 21

- arteries 170, 430

- artery disease 122

- - stenting 555

- dissection 561

- endarterectomy 32,587
Carotid plaques 32

- stenosis 21,555

- ultrasonography 170

Cerebral blood flow 348

- - vessels 430

- hemodynamics 348

- hemorrhage 87

- infarction $87,176,316$

- -, subcortical 297

- infarcts 74

- ischemia 587

Cerebrovascular accident 95

- disease, risk factors 366

Chlamydia pneumoniae 170

Chronic stroke 115

Cigarette smoking 517

Clinical management of stroke 202

Clopidogrel 40, 344

Cognition 59

Computed tomography 144, 241

Contrast-enhanced MR venography 217

Coronary disease 136

CT angiography 144

Danqi Piantan Jiaonang, stroke 450

Delayed ischemic neurological deficits 164

Dementia 234, 324

Diffusion tensor imaging 209

Diffusion-weighted MRI 297

Doxycycline 157

Dural arteriovenous fistulas 304

- sinus thrombosis 217

- sinuses 217

DWI 511

Educational measures 457

Elderly 129

EMG-triggered neuromuscular stimulation 115

Epidemiology 366

Epilepsy 348

Ethanol 517

Ethnicity 408

FAST trial 533

Fibrin 136

Fibrinogen 136

Fibrinolysis 107

Flow dynamics 430

Fractures 95

Functional recovery 423

\section{KARGER}

두 2008 S. Karger AG, Basel

Fax +41 613061234

E-Mail karger@karger.ch

www.karger.com 
Gene polymorphism(s) 81,87

Genetic risk factor 445

Genetics 561

Glucose 136, 261

Glutathione peroxidase1 445

GVS-111 5

Health-related quality of life 67

Hemorrhagic stroke 517

- transformation 241

History of stroke 526

Human platelet alloantigens 81

Hyperbaric oxygenation 193

Hyperperfusion 580

Hypertension 202, 408

Hyponatremia 164

\section{Imaging 457}

Infarct location 100

Infection 50

Inflammation 50, 417

Intima-media thickness 122

Intra-arterial thrombolysis 401, 438, 511

Intracerebral hemorrhage 241, 254, 445, 533

Intravenous thrombolysis 129

Isch(a)emia 5, 50

-, transient 193

Ischemic stroke 26, 40, 129, 170, 241, 254, 316, 338, 417, 517

Lacunar stroke 26

Leukocytes 50

Levetiracetam 5

Lifestyle factor 526

Longitudinal 311

- study 115

Long-term care insurance 234

- mortality 26

- survival 26

Lupus 348

Lymphotoxin $\alpha 417$

Magnetic resonance angiography 430

- - imaging 50,572

Matrix metalloproteinase- 9587

Mechanical embolectomy 338, 401

Meta-analysis 5, 539, 561

Metabolic syndrome 261, 539

Methyltetrahydrofolate reductase 561

Microembolic signals 348

Middle cerebral artery infarction 176

- _ - pathology 74

- _ - stenosis 261

Minocycline 157

Minor ischaemic stroke 225

- stroke 566

Motor deficit 297

- recovery 115

MR venography 217

MRI 21, 338, 511

Myocardial infarction 225
Neomycin 362

Neurological deterioration 151

- rehabilitation 115

Neuroprotection 5, 268

NIHSS score 401

Nonlinear mapping 392

Nonvalvular atrial fibrillation 289

Noopept 5

Normobaric oxygenation 193

Outcome assessment 423

- modelling 225

- research 438

Oxiracetam 5

Oxygen 193

Oxyhemoglobin 362

Paraoxonase 122

- 287

Patent foramen ovale 111

Phospholipase C 362

Piracetam 5

Platelet aggregation 355, 392

- reactivity 392

- recruitment 392

Polymerase chain reaction 81

Polymorphism 417, 561

Pontine infarction 209

Prediction of outcome 202

Public awareness, stroke risks 385

Quality of hospital care 12

- _ life 438,548

Recombinant tissue plasminogen activator 511

Recurrent stroke 254

Registries 12

-, stroke 423

Rehabilitation 457, 566

rt-PA 129

Safety studies 450

Secondary degeneration 209

Seizure onset 100

- types 100

SF-36 67

Small-vessel cerebrovascular disease 408

- diseases of the brain 348

Smoking 324

Speech and language therapy 316

Spouses 311

Status epilepticus 100

Steno-occlusive arterial disease 151

Striatum 297

Stroke 5, 81, 87, 95, 234, 311, 324, 344, 366, 438, 517, 539

- care 566

- - programme, patient satisfaction 566

- - , weekend, off-time 332

- epidemiology 548

- outcome 21,423 
Stroke outcomes 12

- prevention 457

- prognosis 202

- risk factors 385

- treatment 268

- unit 67,457

- warning signs 385

Stroke-related seizures 100

Subarachnoid hemorrhage 144, 164

Superficial temporal artery/middle cerebral artery bypass 580

Systematic review 5

Telemedicine 332

Telestroke 332

Territorial infarcts 100

Thrombolysis $332,338,511$

-, time window 572

Thromboxane $\mathrm{A}_{2} 362$

Ticlopidine 40

Time window admission 533

Time-resolved MR angiography 217

Tissue factor 32

- plasminogen activator 107
TOF MR venography 217

Tolerability 157

Traditional Chinese medicine 450

Transcranial color Doppler flow imaging 304

- _ - sonography 555

- _ ultrasound 111,348, 430

Transient ischaemic attack 225, 566

Type 2 diabetes mellitus 261

Vascular cognitive impairment 408

Vasculostatic therapy 157

Vasomotor reactivity 555

Vasospasm 144, 362

Venous to arterial circulation shunt 111

Visual scale 247

Volumetry 247

White matter 408

- - hyperintensities 59

- $\quad$ - progression 247 